\title{
The Use of Bone-Borne Distractor for Correcting the Maxillary Transverse Discrepancy in an Adult Patient
}

\author{
Victor Prado Curvêllo, ${ }^{1}$ Fabrício Pinelli Valarelli, ${ }^{2}$ Luiz Nunes Pegoraro, ${ }^{1}$ Rodrigo Hermont Cançado,, , \\ Thaís Marchini Oliveira, ${ }^{3}$ and Karina Maria Salvatore de Freitas ${ }^{2}$ \\ ${ }^{1}$ Hospital for Rehabilitation of Craniofacial Anomalies-USP (HRAC-USP), University of São Paulo, Bauru, Brazil \\ ${ }^{2}$ Department of Orthodontics, Ingá Faculty, Maringá, Brazil \\ ${ }^{3}$ Department of Pediatric Dentistry, Orthodontics and Public Health, University of São Paulo, Bauru, Brazil \\ "Corresponding author: Rodrigo Hermont Cançado, Department of Orthodontics, Ingá Faculty, Maringá, Brazil. Tel/Fax: +55-4430335009. E-mail: rohercan@uol.com.br
}

Received 2016 February 06; Accepted 2016 April 23.

\begin{abstract}
Introduction: The objective of this study was to report the correction of a maxillary transverse discrepancy in an adult patient using Le Fort I osteotomy procedure associated with a bone-borne maxillary distractor device. Both the indications, advantages of the procedure and the use protocol were highlighted.

Case Presentation: The results showed that the bone-borne distractor promoted the correction of maxillary transverse discrepancy with minimal side effects on the maxillary posterior teeth.

Conclusions: The bone-borne maxillary distractor device is a good alternative for correcting the maxillary transverse discrepancy in patients undergoing Le Fort I surgery, especially in cases presenting either periodontal disease or gingival recession of maxillary posterior teeth.
\end{abstract}

Keywords: Palatal Expansion Technique, Orthodontic Appliances, Osteotomy

\section{Introduction}

In adult patients, the correction of skeletal maxillary transverse discrepancy is a great challenge mainly related to the treatment stability and side effects on teeth and surrounding structures coming from the expansion protocol (1).

Because adults show great development and maturation of facial bones and increased midpalatal suture interdigitation, surgically-assisted rapid palatal expansion has been indicated in cases of adult patients presenting skeletal maxillary transverse discrepancy (2).

Notwithstanding, conventionally, the expansion screws are supported on the maxillary posterior teeth (molars and premolars) and even if the surgically-assisted expansion procedure releasing the palatal suture and weakening the vertical pillars both the teeth and surrounding tissues may undergo side effects and sequelae. Accordingly, these side effects occur mainly in teeth and tissues previously exhibiting an impaired condition (2).

Thus, to avoid such complications, many bone-borne distractors have been developed (3-5). These distractor devices are directly placed onto the palatal bone during the surgical procedure aiming to guide the applied forces and decrease the injuries caused by the expansion protocol such as: tooth inclination, root resorptions, periodontal ligament compression, fenestration of the buccal bone cortical, and severe bone resorptions $(6,7)$.

This study aimed to report a clinical case of surgicallyassisted rapid maxillary expansion by bone-borne palatal distractor in an adult patient showing severe maxillary atresia associated with mild buccal gingival recessions in maxillary posterior teeth, evidencing the main indications, advantages and activation protocol.

\section{Case Presentation}

Patient A.G.R., aged 38 years, searched treatment with main complaint of anterior open bite and difficult in chewing. At extraoral examination, patient presented a concave profile and passive lip sealing (Figure $1 \mathrm{~A}-\mathrm{C}$ ).

The atresia of the palate showed great severity (Figure $3 A-B)$. During anamnesis, the patient did not report any systemic disease, chronic use of medicines or addictions.

The radiographs showed normal anatomic structures. The mandibular second molar (tooth 37) was absent, the maxillary and mandibular posterior teeth were restored 

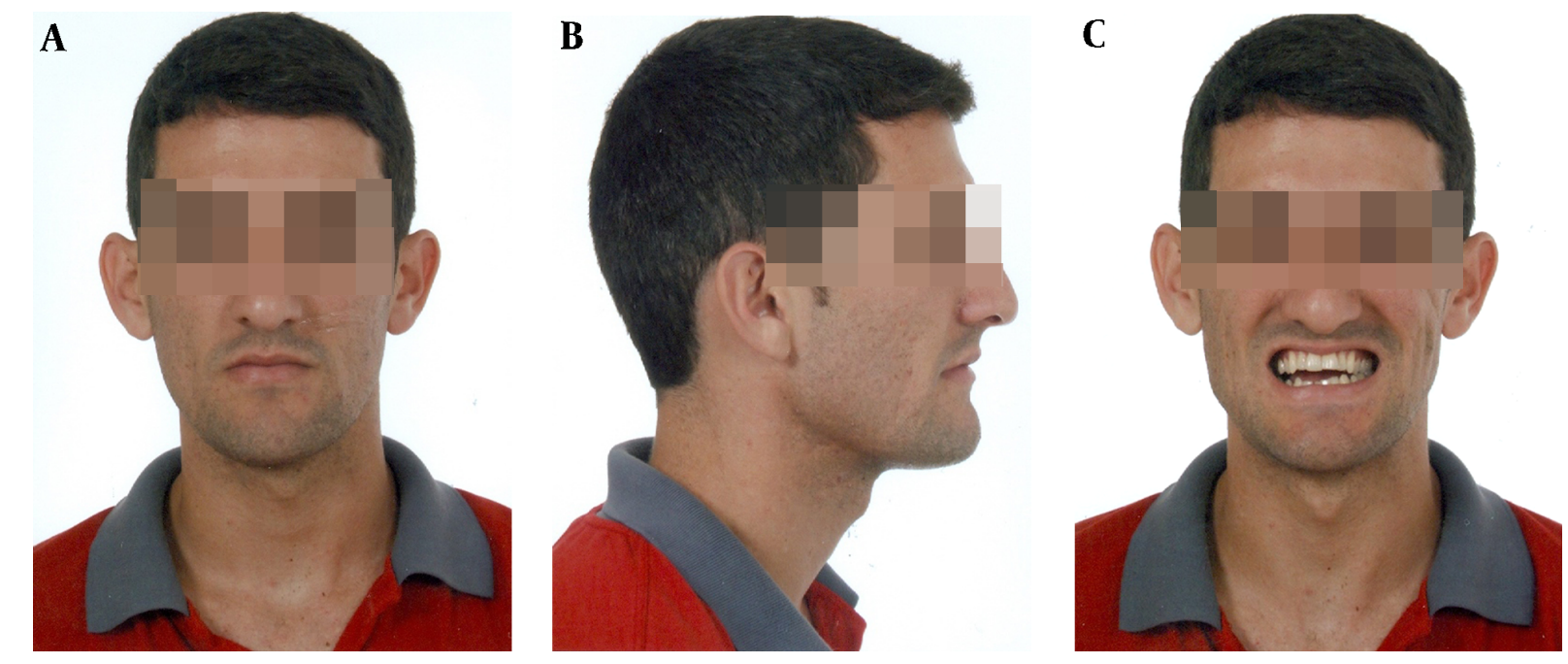

Figure 1. A-C-Extraoral Aspects at the Beginning of Treatment
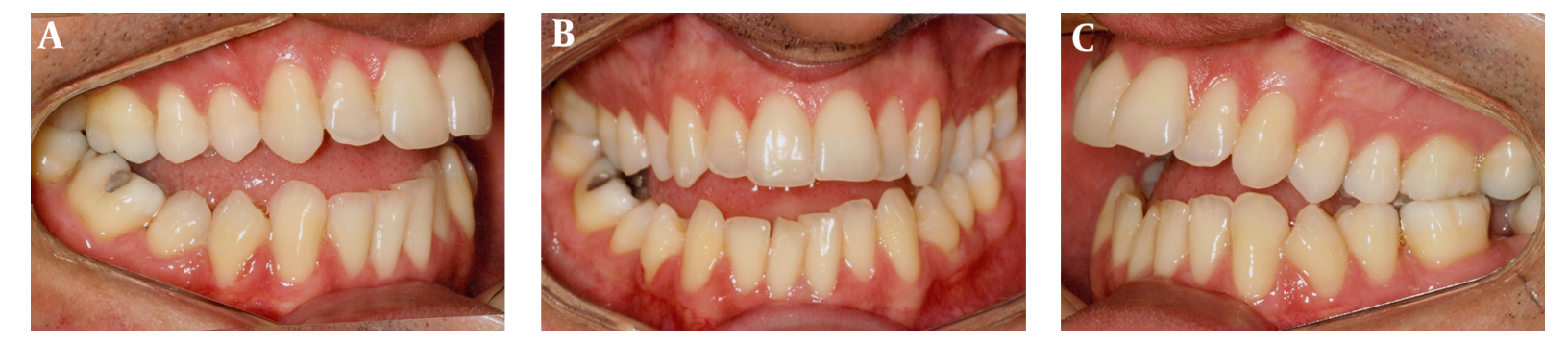

Figure 2. A-C-Intraoral Aspects at the Beginning of Treatment
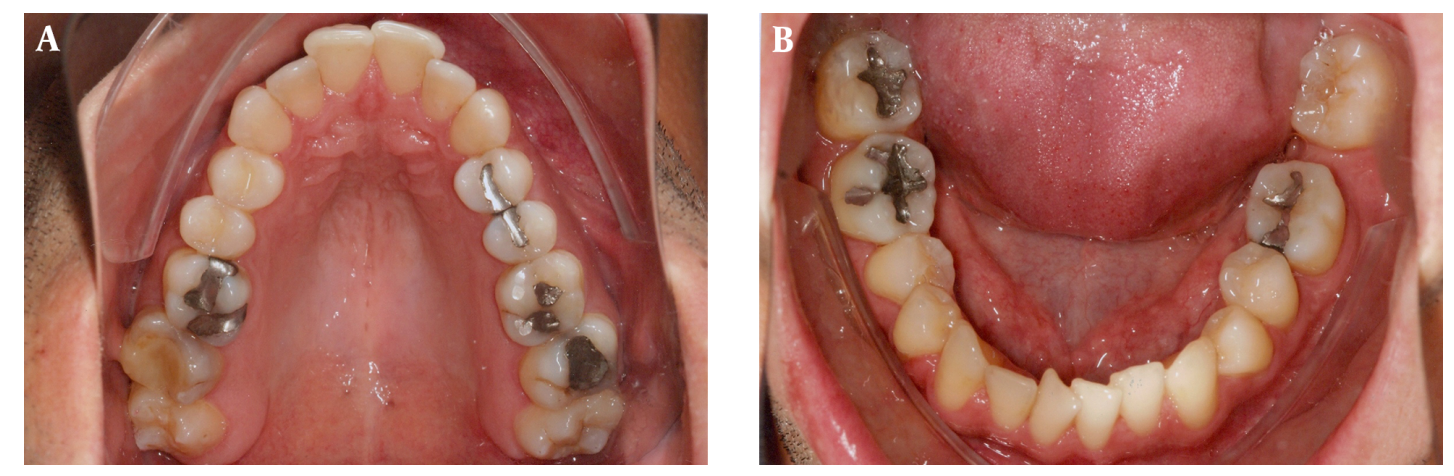

Figure 3. A-B- Occlusal Aspects at the Beginning of Treatment

and the maxillary and mandibular third molars were present (Figure 4).

Considering the initial clinical and radiographic aspects, two treatment alternatives to correct the maxillary transverse discrepancy were planned. The first option comprised the Le Fort I surgery to release the midpalatal suture and maxillary expansion through conventional distractor device (HAAS or Hyrax).

The second option was composed by the Le Fort I surgery to release the midpalatal suture and maxillary ex- 


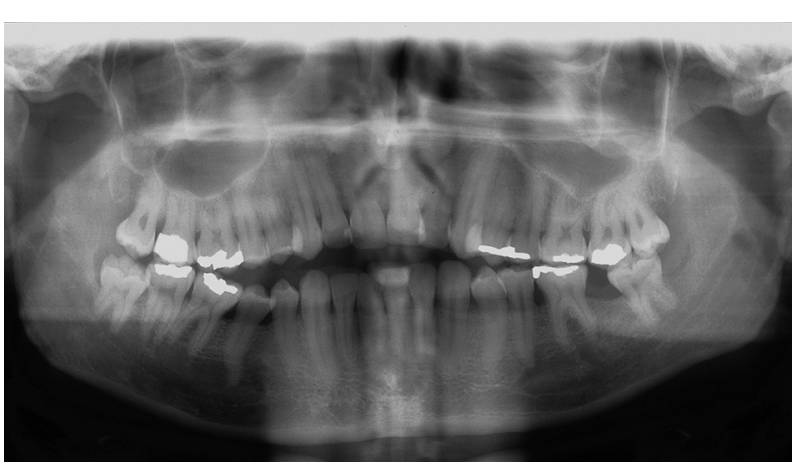

Figure 4. Panoramic Radiograph at the Beginning of Treatment

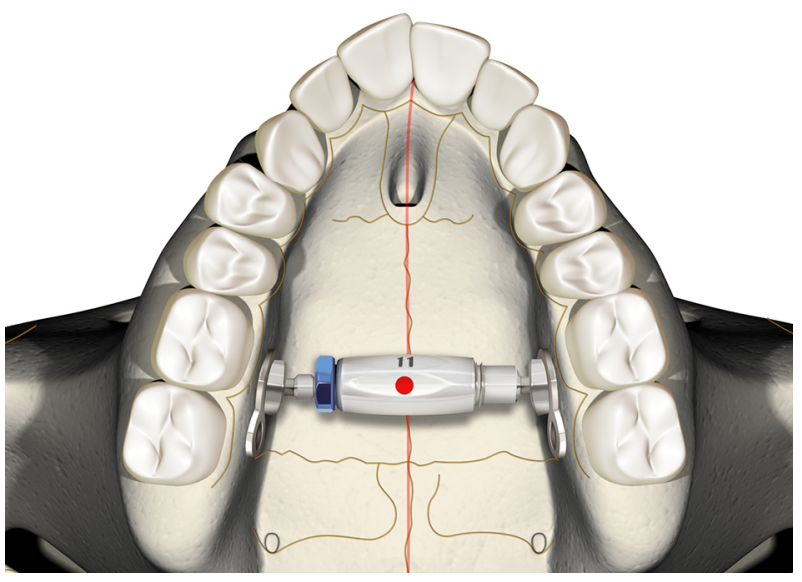

Figure 5. Drawing Illustrating the Bone-Borne Distractor Device Placed on the Maxilla

pansion through bone-borne distractor device (Smile 3 NIVAA, São Bernardo do Campo, São Paulo, Brazil) aiming at preserving the maxillary posterior tissues and structures (Figure 5).

The expansion was carried out at hospital environment, under general anesthesia, through nasotracheal intubation. The fixing screw of the distractor was installed between the roots of teeth 14 and 15, and 24 and 25, through an incision on the palatal area through total flap. With the aid of a template, the 14-mm distractor was chosen, installed with the locker nut placed on the patient's left side, far from the mucosa.

Next, the maxillary vertical bone pillars were released. The incision on the alveolar mucosa of the buccal side was carried out with the aid of electrocautery and dissector tip. The alveolar mucosa flap was raised, and the Le Fort I osteotomy was carried out. After the bilateral releasing of the canine, zygomatic, and pterygomaxillary pillars, the midpalatal suture was released. This latter was carried out with the aid of a mark on the maxillary area below the anterior nasal spine and straight chisel (Figure 6).

After the releasing of the vertical pillars and midpalatal suture, the distractor was activated. This procedure is performed to assure the complete releasing of the midpalatal suture by means of the diastema opened between the maxillary central incisors. After the confirmation of the midpalatal suture releasing, the flap was positioned with continue sutures (polyglactin 910, Johnson \& Johnson, São Paulo, São Paulo, Brazil).

Elapsed 5 post-surgical days, the bone-borne distractor was activated with the aid of specific key so that each activation corresponded to $0.3 \mathrm{~mm}$ (Figure 7).

The activation was performed twice per day, at the morning and night periods. By reaching the overcorrection of the posterior crossbite, which in this patient was of $12 \mathrm{~mm}$, the stability nut was locked at the desired position and prevented the closure of the screw (Figure 8). At that moment, an oclusal radiograph of the maxilla was taken to assess the radiographic characteristics of the maxillary disjunction (Figure 9).

After a 6-month period to obtain the bone repair on the midpalatal suture, this was confirmed by the occlusal radiograph (Figure 10). At that stage, the alignment and leveling of the maxillary and mandibular teeth was initiated followed by the orthodontic treatment to correct the malocclusion (Figure 11).

\section{Discussion}

The maxillary expansion is part of the orthodontic therapy armamentarium aiming at treatment success in cases of transverse discrepancy. In adult patients, this approach might result in undesirable side effects for the teeth and surrounding structures, thus, the surgery of releasing of vertical pillars and midpalatal suture has been well indicated in these cases (8).

However, even with the releasing of bone resistance, the traditional expansion devices are supported by the teeth, frequently molars and premolars (6). This characteristic increases the risks of side effects on teeth and surrounding structures, mainly when these tissues already showed some degree of impairment before the procedure (6).

A good choice for adult patients is the bone-borne distractor because of the exclusively orthopedic maxillary movement which gives great predictability to the maxillary expansion by applying the forces directly on the bone without undesirable effects on teeth and periodontal ligament (9-11).

The bone-supported rapid maxillary expansion provides great expansion at the anterior area, thus with 

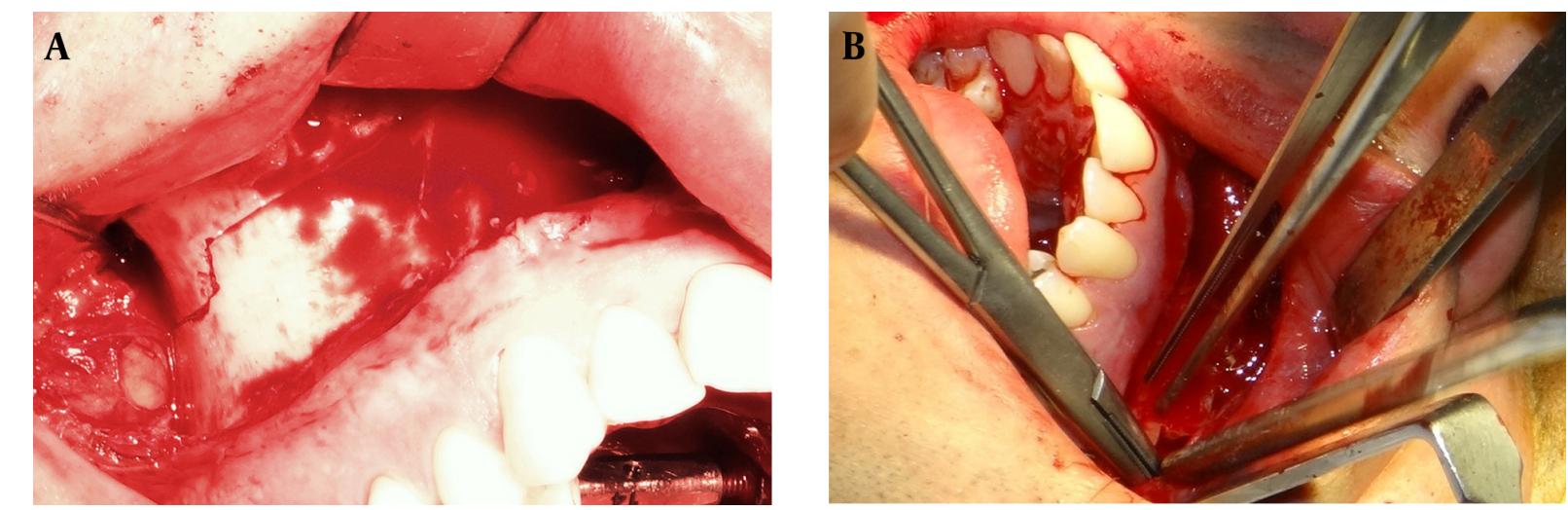

Figure 6. Osteotomy of the Maxillary Vertical Pillars

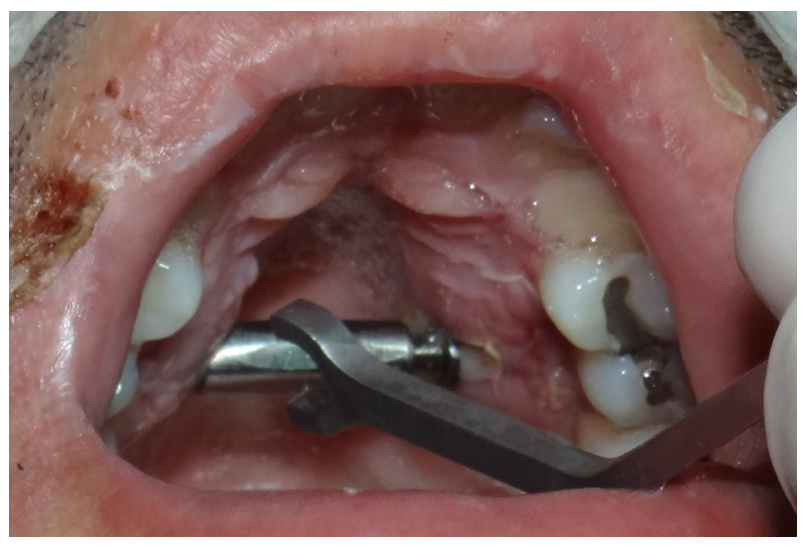

Figure 7. Bone-Borne Distractor Activation

greater effectiveness to the procedure (11). The transmission of the screw expansion to the alveolar bone is greater in bone-supported than in tooth-supported rapid maxillary expansion $(11,12)$.

Although in bone-supported rapid maxillary expansion (BSRME), the tooth expansion pattern follows the midpalatal suture expansion, the transverse increase of the molars is 0.6 times greater, in average, that of canines, while in the surgically-assisted rapid maxillary expansion (SARME) is of 1.12 times and in rapid maxillary expansion (RME) is of 2.01 times (12). Therefore, BSRME provides greater orthopedic expansion (12) and this effect generates great increasing of the arch perimeter (mean of 10.5\%) mainly due to the increasing of intercanine distance (7). Currently, the literature reports that BSRME provides a bone transverse gain larger than that obtained by traditional tooth-borne expansion devices $(4,11,12)$.

Bone-borne distractor is another tool in the armamen- tarium of the treatment of maxillary transverse discrepancies, considerably decreasing the side effects when compared with the traditional methods $(7,11)$. Moreover, this device has been well indicated for patients exhibiting severe maxillary transverse discrepancy together with previous impairment of teeth and surrounding tissues in the maxillary posterior area (13).

In the clinical case presented here, the bone-supported expansion promoted excellent results, favoring the increasing of arch perimeter for tooth alignment and maintaining stable the levels of gingival recessions of the maxillary posterior teeth showed before treatment.

At every year, new devices are launched in dental marked aiming to improve the science of osteogenesis through distraction to correct the transverse discrepancy. The device used in this case report tis biocompatible, costeffective, reliable, and safe. The surgery for its installation and removal is also very simple and it favors oral hygiene due to its "modern design" with little discomfort to patient.

\subsection{Conclusions}

Based on the results of the procedure presented in this clinical case report, the bone-borne distractor is a good option for correcting the maxillary transverse discrepancy providing little side effects on the teeth and surrounding tissues.

\section{Footnotes}

Authors' Contribution: Victor Prado Curvêllo developed the original idea and the protocol and wrote the manuscript. Fabrício Pinelli Valarelli prepared and wrote the manuscript and is guarantor. Luiz Nunes Pegoraro contributed to the development of the protocol, abstracted 

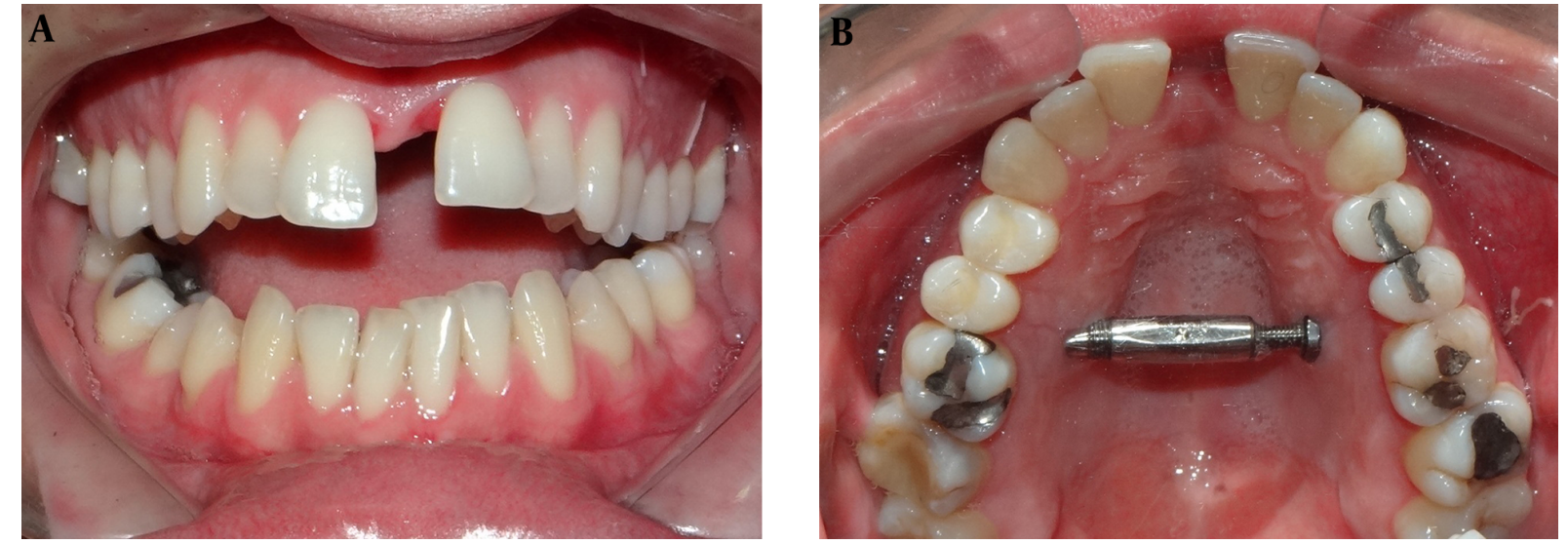

Figure 8. Photographs at the Ending of the Distractor Activation

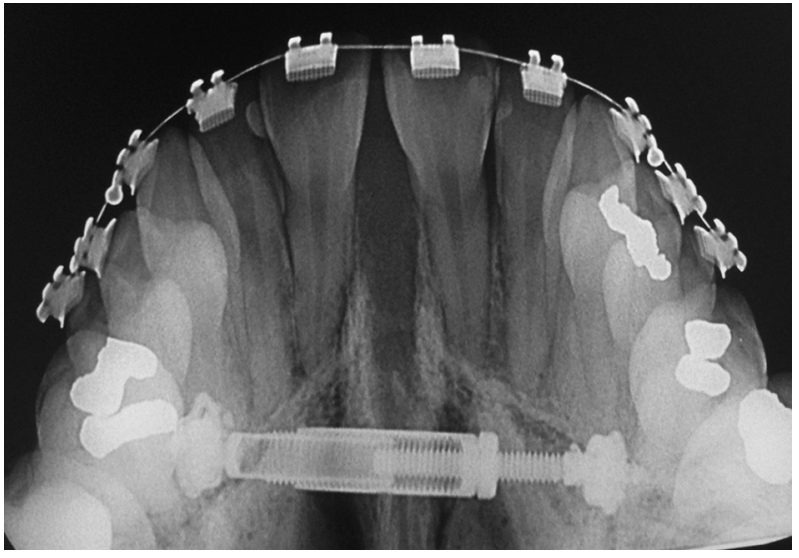

Figure 9. Opening of the Midpalatal Suture on the Oclusal Radiograph

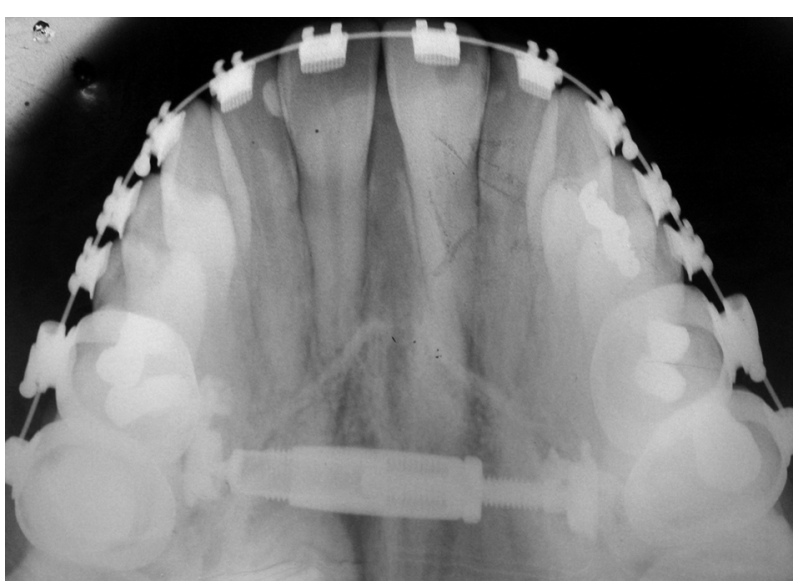

Figure 10. Maxillary Occlusal Radiograph at 6-Month Post-Surgical Period

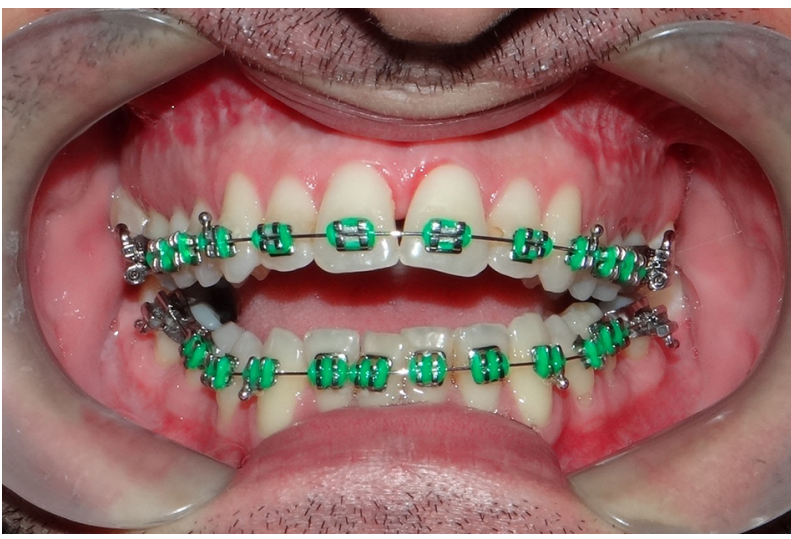

Figure 11. Intraoral Aspect of the Alignment and Leveling Phase

data, and prepared the manuscript. Rodrigo Hermont Cançado wrote the manuscript, contributed for the critical revision of the manuscript for important intellectual content. Thais Marchini Oliveira wrote the manuscript, contributed for the critical revision of the manuscript for important intellectual content.

Financial Disclosure: Marring \& acute; February $2^{\text {nd }}$, 2016. Re: Conflict of interest, regarding the article \& liquor; the use of bone-borne distractor for correcting the maxillary transverse discrepancy in an adult patient \& rdquo; we do not have a significant financial or professional interest in any company, product, or service mentioned in the article. Sincerely, Dr. Rodrigo Hermont Cançado (Corresponding author) 


\section{References}

1. Bell WH, Epker BN. Surgical-orthodontic expansion of the maxilla. Am J Orthod. 1976;70(5):517-28. [PubMed: 790971].

2. Betts NJ, Vanarsdall RL, Barber HD, Higgins-Barber K, Fonseca RJ. Diagnosis and treatment of transverse maxillary deficiency. Int J Adult Orthodon Orthognath Surg. 1995;10(2):75-96. [PubMed: 9082002].

3. Garib DG, Navarro RL, Francischone CE, Oltramari PVP. Expansão rápida de maxila ancorada em implantes. Uma nova proposta para a expansão ortopédica em pacientes. Dental Press Ortodon Ortop Facial. 2007;12:75-81.

4. Gunbay T, Akay MC, Gunbay S, Aras A, Koyuncu BO, Sezer B. Transpalatal distraction using bone-borne distractor: clinical observations and dental and skeletal changes. $J$ Oral Maxillofac Surg. 2008;66(12):2503-14. doi: 10.1016/j.joms.2008.06.105. [PubMed: 19022131].

5. Koudstaal MI, van der Wal KG, Wolvius EB, Schulten AJ. The Rotterdam Palatal Distractor: introduction of the new bone-borne device and report of the pilot study. Int J Oral Maxillofac Surg. 2006;35(1):31-5. doi: 10.1016/j.ijom.2005.07.002. [PubMed: 16154318].

6. Matteini C, Mommaerts MY. Posterior transpalatal distraction with pterygoid disjunction: a short-term model study. Am J Orthod Dentofacial Orthop. 2001;120(5):498-502. doi: 10.1067/mod.2001.118401. [PubMed: 11709668].

7. Pinto PX, Mommaerts MY, Wreakes G, Jacobs WV. Immediate postexpansion changes following the use of the transpalatal distractor. $J \mathrm{Am}$
Asso Oral Maxillofacial Surgeons. 2001;59:994-1000.

8. Kraut RA. Surgically assisted rapid maxillary expansion by opening the midpalatal suture. $J$ Am Asso Oral Maxillofacial Surgeons. 1984;42:651-5.

9. Harrison JE, Ashby D. Orthodontic treatment for posterior crossbites. Cochrane Database Syst Rev. 2001(1):000979. doi: 10.1002/14651858.CD000979. [PubMed: 11279699].

10. Petren S, Bondemark L, Soderfeldt B. A systematic review concerning early orthodontic treatment of unilateral posterior crossbite. Angle Orthod. 2003;73(5):588-96. doi: 10.1043/00033219(2003)073<0588:ASRCEO>2.0.CO;2. [PubMed: 14580028].

11. Tehranchi A, Ameli N, Najirad Z, Mirhashemi FS. Comparison of the skeletal and dental changes of tooth-borne vs. bone-borne expansion devices in surgically assisted rapid palatal expansion: A finite element study. Dent Res J(Isfahan). 2013;10:777-83.

12. Tausche E, Hansen L, Hietschold V, Lagravere MO, Harzer W. Threedimensional evaluation of surgically assisted implant bone-borne rapid maxillary expansion: a pilot study. Am J Orthod Dentofacial Orthop. 2007;131(4 Suppl):92-9. doi: 10.1016/j.ajodo.2006.07.021. [PubMed: 17448393].

13. Iida S, Haraguchi S, Aikawa T, Yashiro K, Okura M, Kogo M. Conventional bone-anchored palatal distractor using an orthodontic palatal expander for the transverse maxillary distraction osteogenesis: technical note. Oral Surg Oral Med Oral Pathol Oral Radiol Endod. 2008;105(2):8-11. doi: 10.1016/j.tripleo.2007.08.022. [PubMed: 18230382]. 